\title{
PERCEPCIONES SOBRE EL ESTUDIO DE CASOS, COMO ESTRATEGIA DE APRENDIZAJE, EN ESTUDIANTES DE ENFERMERÍA
}

\author{
PERCEPTIONS ABOUT THE CASE STUDY, AS LEARNING \\ STRATEGY IN NURSING STUDENTS
}

\author{
María Patricia González Vega* \\ JAVIER VERGARA NúÑEZ ${ }^{* *}$
}

\begin{abstract}
RESUMEN
La presente investigación de enfoque cualitativo, basada en la compilación de información mediante la observación en el aula y sistematizada a través de algunos métodos e instrumentos derivados de la teoría fundamentada, tuvo como objeto analizar las percepciones de estudiantes de Enfermería sobre el 'estudio de casos', estrategia de aprendizaje utilizada por la carrera, en la Universidad del Mar. A partir de la información reunida se elaboraron categorías interpretativas, con el fin de comprender y explicar el valor pedagógico del proceso didáctico en estudio. Los sujetos investigados corresponden a un grupo de estudiantes de segundo año de la carrera, con quienes, uno de los docentes investigadores, trabajó la estrategia de aprendizaje, 'estudio de casos'. La información fue obtenida a través de: la observación participante, las entrevistas semiestructuradas, el grupo de discusión y la revisión de documentos. De los resultados obtenidos emergieron las siguientes 'categorías': aprendizaje, trabajo en equipo, mediación docente y formación profesional. Como conclusiones, los estudiantes revelaron que, mediante el 'estudio de casos', experimentaron un proceso fundamental en su formación profesional: fortalecer el trabajo en equipo y desarrollar cualidades para liderar un grupo. También haber desarrollado importantes cambios en la forma de aprender, transformándose en protagonistas activos que toman sus propias decisiones y que construyen aprendizajes significativos en la aplicación de situaciones reales, perfilando un rol profesional sólido que le permitirá un buen desempeño en la solución de problemas de su quehacer profesional.
\end{abstract}

Palabras clave: Estudio de casos, percepción, estudiantes de Enfermería, aprendizajes, educación profesional.

\begin{abstract}
The purpose of this research with a qualitative approach, based on information compiled during classroom observation and systematized through some methods and tools derived from grounded theory, was to analyze the perceptions of nursing students about the 'case study' learning strategy used by the nursing career, at the Universidad del Mar. In order to understand and explain the pedagogical value of the didactic process in study, interpretative categories were elaborated from the gathered information. The investigated subjects are a group of nursing students in their second year, with whom, one of the academic researcher, worked out the learning strategy. The information was gathered through: participant observation, structured interviews, group discussions and document review. From the reached results, the following 'categories' emerged: learning, teamwork, tutorial mediation and vocational training. Conclusion, the students revealed that, through 'case study', they experienced a fundamental process in their professional training: strengthening teamwork and developing skills to lead a group. They also recognize important changes in the way of learning, becoming active students making their own decisions and who construct significant learning in the application of real
\end{abstract}

\footnotetext{
*Enfermera, docente Universidad del Mar, Viña del Mar Chile, Email: mariapatricia.gonzalez@udelmar.cl

** Profesor de Historia y Geografía, docente de la Universidad de Playa Ancha, Valparaíso, Chile. Email: javier.vergara.n@ gmail.com
} 
situations, outlining a strong professional role that will allow a good performance in solving their professional problems at work.

Key words: Study of cases, perception, students, Nursing, learning, education, professional.

Fecha recepción: 21/03/11 Fecha aceptación: 09/12/11

\section{INTRODUCCIÓN}

Esta investigación pretende ser una contribución a la discusión de los paradigmas didácticos utilizados en la educación superior, sistema que en todos sus niveles enfrenta cambios en la forma como enseñan los profesores, y de como aprenden los estudiantes. Los docentes universitarios deben promover el uso de estrategias de aprendizaje enmarcadas en estas nuevas tendencias, procurando adquirir conocimientos que promuevan el desarrollo de un profesional con sólidas competencias (1). Se entiende por estrategias de aprendizaje a aquellas actividades que realiza el estudiante para facilitar la adquisición de conocimiento (2). Las estrategias de aprendizaje comprenden todos aquellos recursos cognitivos que utiliza el estudiante para el logro del aprendizaje, incorporando además, elementos vinculados a las actividades de planificación, dirección y control cuando se enfrenta a una tarea. Todo este proceso forma parte de un funcionamiento estratégico que podría garantizar la obtención de aprendizajes altamente significativos. Una de las estrategias educativas que ha revelado poseer características idóneas para el aprendizaje, es el 'estudio de casos, " “...que tiene como objeto la capacitación práctica para la solución de problemas. Analizarlos y reflexionar sobre ellos constituye un ejercicio mucho más importante que hallar la solución. Ayuda a aprender a considerar las opiniones de los compañeros y admitir que se pueden dar varias soluciones correctas para un mismo problema. Es un método de formación en profundidad, que genera actitudes favorables, conduce al análisis metódico y entrena para una correcta toma de decisiones" (3).

Algunas investigaciones examinadas en el ámbito de la educación describen resultados alcanzados tras la aplicación del 'estudio de casos', principalmente en asignaturas del área de las ciencias. Lewis (4) señala que el profesor necesita incorporar estrategias como el 'estudio de casos', lo que permite desarrollar habilidades de pensamiento a altos niveles. Herreid (5) advierte que la enseñanza con estudio de casos, sitúa a los alumnos en los problemas del mundo real y los desafía a pensar cada uno de los pasos para su solución. Ribbens (6) discute que es esencial enseñar a los estudiantes a manejar la información, a tomar decisiones basadas en la evidencia, a desarrollar modelos conceptuales, y a declarar la calidad y relevancia de una información en un problema concreto. Gallucci (7) identificó tres principios acerca de cómo los estudiantes aprenden. Primero, se debe encauzar los conocimientos previos de los alumnos. Segundo, se debe proveer una estructura conceptual de conocimiento, $y$, tercero, se debe entrenar a los estudiantes a usar estrategias metacognitivas, es decir, los estudiantes deben aprender a dirigir y tomar control de su propio aprendizaje. El método de 'instrucción de casos' puede ser usado para direccionar estos tres principios. Malkani y Allen (8) sugieren que las actividades de aprendizaje reflexivo, como los casos, ayudan a desarrollar habilidades de pensamiento crítico y reflexivo de los estudiantes en práctica. 
Siendo esta estrategia una oportunidad de reflexión y de aprendizaje significativo, en que el estudiante asume un papel fuertemente protagónico, se hace necesario observar y describir las propias impresiones de lo que él hace, de cómo se comporta, de cómo interactúa, en qué cree, qué valores lo representan, cuáles son sus motivaciones, expectativas y sentimientos en los diferentes momentos y circunstancias a los que se ve enfrentado, mientras avanza en el trabajo de desarrollar y decidir las soluciones de un caso. El estudiar estas percepciones podría servir para identificar posibles debilidades y amenazas que surgen de la aplicación de este método, pudiendo constituir un aporte de mejoras al momento de su implementación, y resguardar los beneficios académicos que el método propone. Aunque sobre el fenómeno investigado: el 'estudio de casos', se ha detallado una amplia exploración acerca de sus atributos pedagógicos, poco se ha analizado desde un punto de vista interpretativo, las percepciones que tienen los estudiantes en el terreno formativo de la Enfermería. En concordancia con lo anterior, el objetivo del estudio es analizar las percepciones que los estudiantes de la carrera de Enfermería tienen acerca del uso del 'estudio de casos'. La presente investigación se enmarcó en un paradigma interpretativo, de carácter cualitativo (9), con un diseño derivado de los métodos (10-11) propuestos por la teoría fundamentada, cuidando de mantener una relación directa y cercana a los actores del espacio social en que se situó. El investigador se familiarizó con el quehacer de los sujetos implicados en el trabajo, para luego iniciar el análisis y la interpretación de las percepciones más relevantes de los estudiantes respecto al 'estudio de casos'. Es así como se logró analizar y describir las ideas y creencias de un grupo de estudiantes de Enfermería, comprendiendo desde dentro el fenómeno educativo en estudio y explicando la realidad en base a la percepción de los aprendices.

\section{MATERIAL Y MÉTODO}

Esta investigación, como se indicó previamente, se desarrolló en el marco de un paradigma interpretativo, de carácter cualitativo, que utilizó para la compilación y tratamiento de la información algunos métodos inspirados en la Teoría Fundamentada, como lo son la observación del trabajo realizado por los estudiantes en el aula, las entrevistas y el diálogo mediado por el investigador, además del análisis de algunas reflexiones escritas por los estudiantes, elaboradas durante el proceso formativo y transcritas en un portafolio grupal, que se utilizó como método evaluativo. Los sujetos seleccionados para la investigación son parte de un curso en el que se aplicó la estrategia de aprendizaje, 'estudio de casos', cuestión que constituyó el criterio fundamental de inclusión. Corresponde a 24 estudiantes, hombres y mujeres, entre 19 y 27 años de edad, de segundo año de la carrera de Enfermería, de la Universidad del Mar, de la Región de Valparaíso. Para la recolección de la información se llevaron a cabo los siguientes métodos: Observación participante (12), en que el investigador realizó la inmersión de campo con un rol de facilitador y mediador docente(13) en uno de los grupos de trabajo de los estudiantes, en su ambiente educativo natural, estableciéndose una relación de interacción personal que posibilitó el trabajo académico, situación que incentivó el diálogo, la discusión y el intercambio de experiencias sobre las problemáticas analizadas y el proceso formativo. Entrevistas semiestructuradas, en que se realizaron preguntas abiertas, que orientaron la obtención de la información, expresada en las respuestas verbales de los sujetos, las que fueron grabadas y registradas para su análisis posterior. Grupo de discusión (14), el que se constituyó con los estudiantes investigados, con el objeto de revelar, mediante una discusión libre (a través de una consigna centrada en el objeto investigado), las experiencias, percepciones y 
valoraciones que fueron vivenciando y elaborando acerca de la propuesta de la estrategia de aprendizaje en estudio. Análisis de documentos, que fueron constituidos por reflexiones acerca de la experiencia de desarrollar un caso. Estas fueron documentadas de forma individual y grupal por los propios estudiantes. Estos escritos eran parte de un portafolio que fue confeccionado como apoyo evaluativo.

El plan general de análisis se estructuró a partir de la estrategia metodológica de profundización progresiva del conocimiento del objeto estudiado. Se inicia la investigación con la observación natural en el aula, de ello se derivan algunas interrogante que, en una segunda fase, se clarifican a partir de la observación participante en el grupo de trabajo de los alumnos. Luego, en una tercera fase, se logra un mejor entendimiento con las entrevistas semiestructuradas. Tras ello, en una cuarta fase, se verifica la información, abriendo con un grupo de discusión, un diálogo más amplio; esta técnica se implementó como alternativa de clarificación de algunos conceptos que se encontraban aún difusos para los investigadores y, finalmente, en una quinta fase, se procedió al análisis de las producciones de los estudiantes (portafolios), analizando el registro de sus opiniones y comentarios sobre el uso del método 'estudio de casos', verificando y profundizando, con esto, la comprensión de la información compilada, de lo observado al natural, además de lo expresado tanto en la observación participante, las entrevistas, como en el grupo de discusión.

Para el resguardo del rigor y la fiabilidad del análisis de la información, tanto en la fase compilatoria como en la de tratamiento, se desarrolló una observación continua de las clases en donde se aplicaba la estrategia de aprendizaje 'estudio de casos'; luego se recogió fielmente las verbalizaciones de los estudiantes, durante la observación participante, en las entrevistas y en los grupos de discusión, con registro escrito y magnetofó- nico, contrastando finalmente, el conjunto de la información recogida, con el análisis exhaustivo de las producciones escritas de los estudiantes. Se constituyó un corpus de información abundante y variada sobre el objeto en estudio, en momentos distintos, al que se le aplicó un análisis progresivo (en amplitud y profundidad), a modo de captar la complejidad y multivariada dimensión del objeto en estudio, cuidando en cada fase la independencia del análisis, a través de investigadores externos.

Con el interés de alcanzar el objetivo central de esta investigación, se estudiaron todas las percepciones que los estudiantes de Enfermería elaboraron acerca del 'estudio de casos', como estrategia de aprendizaje. Esta información fue analizada utilizando el método de Análisis de Contenido. Primeramente en general se realiza una lectura completa de la información obtenida y luego, en particular emergen agrupaciones de unidades de análisis, en 'categorías' y 'subcategorías' definitivas (15), descritas más adelante en los resultados. Para una mejor comprensión de ellas, se establecieron cuatro 'categorías', que corresponden a las menciones de mayor globalidad. Derivadas de las anteriores, se definieron 'subcategorías', considerando que solo la Categoría 2 ofrece la posibilidad de una mayor variación del desarrollo interpretativo.

La participación de los estudiantes en el estudio se certificó en un Consentimiento Informado, resguardando el anonimato y la confidencialidad de la información entregada, la que podrá ser utilizada sólo para la difusión científica.

\section{RESULTADOS}

Los resultados se presentan a partir de las categorías y subcategorías establecidas, ilustradas con citas textuales de los estudiantes. 
Categoría 1. Equipo de trabajo: La labor en 'equipo' conformado por los estudiantes, constituyó el contexto en el que se llevó a cabo la actividad pedagógica del 'estudio de casos' y, por tanto, la que denota una mayor frecuencia en la expresión de sus percepciones.

“... Fue más fácil hacerlo con mis compañeros que sola" (Sujeto 9)..."A través de un trabajo que esto debe ser en grupo" (Sujeto 4)

No obstante, las siguientes manifestaciones expresan la dificultad inicial de trabajar en grupo con personas con las que no habían tenido la oportunidad de relacionarse.

"Yo no sabia si ellos iban a hacer las cosas..." (Sujeto 7)

"Todavía no nos acostumbramos a trabajar con otros compañeros, porque no conocemos la forma en que trabajan" (Documento de reflexiones, grupo 1)

“...debería ser con personas que uno confie, persona que uno 'haiga' trabajado ya antes... pero cuando es personas nuevas es súper difícil porque hay que ir a empezarla a conocerla, que ellos saben que ellos no saben hacer, entonces eso hace mucho más lento el trabajo" (Sujeto 17)

Sin embargo, trabajando juntos y a raíz de un mayor conocimiento entre pares, los estudiantes remarcan el hecho de haber vencido sus diferencias $y$, no obstante las múltiples objeciones en sus grupos de trabajo, señalan haber logrado cumplir con la tarea asignada.

"Estamos contentos porque esto marcha mejor de lo esperado, ya somos un equipo de trabajo, organizado y productivo, podemos salir adelante" (Documento de reflexiones, grupo 4)

“...me quedó la experiencia de trabajar con di- ferentes personas en el cual nunca había tenido un contacto anterior y tener un buen trabajo en equipo y con buenos resultados" (Sujeto 21)

Por otra parte también el estudiante extrapola las habilidades sociales adquiridas al trabajo en equipo del ámbito profesional.

“...poder trabajar en grupo con personas nuevas es una experiencia enriquecedora ya que nos prepara para nuestro futuro como profesionales de Enfermería" (Sujeto 1)

“...cuando uno va a salir a trabajar no sabe con las personas que va salir a trabajar, debe saber adaptarse a ese equipo de trabajo..." (Sujeto 7)

\section{Subcategoría 1.1. Trabajo en equipo cola-} borativo: El estudiante señala que el trabajo en equipo colaborativo es asociado a conseguir mejores resultados académicos.

“...todos pusimos nuestro granito de arena para que este caso integrado resultara lo mejor posible..." (Sujeto 5)

“...todos aportábamos conocimientos... Algunos eran mejor contextualizando, otros redactando, otros haciendo mapas... eso nos facilitó todo" (Sujeto 11)

“...nos ayudábamos entre todos... para imprimir, para corregir, para buscar información..." (Sujeto 13)

"...te falta algo, ya he presta yo te ayudo tu entendí más esta parte dime como hacerla..." (Sujeto 17)

Subcategoría 1.2. Liderazgo: Los estudiantes relacionan el 'liderazgo' al concepto 'equipo de trabajo'.

"Tiene que haber un 'cabecilla' en el grupo, alguien tiene que autoasignarse o que lo asignen..., que sea un líder y que organice el grupo..., el trabajo". (Sujeto 6) 
Esta cualidad la expresan atendiendo a sus propias características personales.

"Trato de dar disponibilidad a mis compañeras..." (Sujeto 7)

“...yo solo daba las ideas,...daba la orden y mis compañeras seguían lo que yo decía”. (Sujeto 10)

“...mis compañeros...si tienen dudas acuden a mí, me preguntan cosas”. (Sujeto 6)

“... tengo una pequeña como etapa de líder, no me es difícil guiar al resto de las personas..." (Sujeto 8)

Categoría 2. Aprendizaje: El 'estudio de casos' es una metodología de aprendizaje, concepto que los estudiantes reconocen como tal.

“... me gusta como que es interesante ver el desarrollo de un problema que se planteó y después ver que primero hay un esqueleto y que después se forma un cuerpo..., es como bien interesante, ...como que uno aprende más" (Sujeto 6)...

"Hemos avanzado con el aprendizaje". (Sujeto 1)

"...pero al final igual uno se da cuenta que aprendió de todo lo que realizó en el trabajo...". (Sujeto 15)

Subcategoría 2.1. Aprendizaje de conocimientos: Los estudiantes aprecian el 'estudio de casos' como herramienta para adquirir diferentes tipos de conocimientos y en profundidad. Esta amplitud temática favorece el aprendizaje individual, en atención a que cada estudiante busca de modo personal clarificar aspectos específicos en campos de estudio igualmente determinados, siguiendo su propia línea de intereses y necesidades formativas. En cuanto a la profundidad que alcanza el aprendizaje, cada estudiante se va transformando en constructor de sus conocimientos y regulador de los ritmos en que los aprende.

"Aprender más, adquirir más conocimientos del caso integrado,... la parte social, el autocuidado, factores de riesgo, la enfermedad en sí, y muchas cosas más" (Sujeto 2)

"Bien, porque existe crecimiento del conocimiento" (Sujeto 6)

“...agrandé mis conocimientos. En cuanto a cantidad y a calidad” (Sujeto 8)

"Como que empieza a tener conocimientos que antes no tenía. Ahora si me preguntan algo de eso yo ya lo voy a saber" (Sujeto 12)

“... además logré incorporar conocimientos y cosa mucho más científicas que es lo que más domino" (Sujeto 19)

Subcategoría 2.2. Aprendizajes de conocimientos integrados: La falta de integración de conocimientos es una dura crítica que reciben las estrategias didácticas; se apunta al hecho de que se deja en manos de los estudiantes la decantación e integración final del saber aprendido, sin las ayudas pedagógicas necesarias. Esto lo constatamos en las verbalizaciones de los estudiantes, quienes declaran haber recibido una formación desintegrada y parcial, respecto a los saberes.

"Me ha ayudado bastante la integración de los ramos antes estudiados, con relacionarlos $y$ desarrollarlos junto a este caso clínico" (Sujeto 6)

"El desarrollo de un trabajo de tipo 'integrador' nos ayuda a reconocer y apreciar todos los conocimientos adquiridos durante nuestro proceso formativo al interrelacionar conceptos, terminologías, y conocimientos en general que determinan la condición de salud o enfermedad en una persona" (Documento de reflexiones, grupo 4) 
“...esto incorpora todo y vamos repasando contenidos básicos como de Biología y Química, hasta ir avanzando en cosas que no manejamos todavía como la Gestión, por ejemplo, incorpora todo el ámbito de la Salud." (Sujeto 13)

“... es una buena forma de aprender ya que se pueden integrar todos los conocimientos adquiridos" (Sujeto 2)

"En cuanto a la elaboración del caso, estoy aprendiendo a integrar todo lo que nos han enseñado..." (Sujeto 3)

También los estudiantes reconocen que con esta forma de aprender, esos conocimientos permanecerán por largo tiempo en sus memorias.

“... los conocimientos van a perdurar más en el tiempo, porque uno no está estudiando de memoria, sino que integrando conocimientos paulatinamente" (Sujeto 2)

"Si uno estudia para una prueba, lo estudié y puede que se me olvide..., pero así no se olvida" (Sujeto 13)

Subcategoría 2.3. Transferencia de los aprendizajes: Un aspecto valioso de la estrategia de aprendizaje implementada, que se revela en la percepción de los estudiantes, lo constituye la apropiación e integración de los aprendizajes, en el hecho de lograr transferir los conocimientos adquiridos a una situación real de contextos específicos.

“... me di cuenta que sí sirve, entiendo la lógica de porqué pasan las cosas que tienen que pasar en cada ramo. No es que es para la prueba, sino que para qué me va a servir" (Sujeto 7)

“...ahí realmente ve la aplicación real de todos los conocimientos que se han adquirido, es como que le ve el uso de lo que uno aprende en el caso del estudio clínico" (Sujeto 6)
"Me gusta lo de aplicar, lo de tener que ver a una persona en una situación y empezar a recordar todo lo que uno ya sabe" (Sujeto 10)

Transferir conocimientos es dar a lo que se aprende un sentido práctico, concreto, efectivo y contextual; este proceso sólo ocurre si los estudiantes comprenden el sentido de cada saber nuevo, si logran producir vínculos integradores entre ellos primero $y$, con los saberes que ya poseían (aprendizajes previos). Las expresiones denotan que efectivamente los saberes son puestos en acción, cualidad que distingue a un profesional competente.

\section{Subcategoría 2.3.1. Aplicación de los apren-} dizajes en la práctica: Paralelo al 'estudio de casos' los estudiantes se encuentran en prácticas clínicas, situación que no ha quedado ajena a su trabajo con el 'caso', estableciendo una conexión entre la práctica y la experiencia de la clase, complementándose y enriqueciéndose mutuamente, según lo denotan sus expresiones:

“...en la práctica profesional se presentaban situaciones parecidas a las relatadas en el trabajo que tengo que realizar, por esto comprendí que es de gran importancia realizar este trabajo debido a que en mi futuro profesional se presentarán, al igual que en la práctica, situaciones análogas..." (Sujeto 4)

"Todos nuestros conocimientos son llevados a la práctica a través de un caso de una persona x" (Sujeto 11)

"Me facilitó entender algunas materias, porque no veía la relación de materias con prácticas no sabía como integrarlos, entonces ahora sé que esto me sirve para esto. $Y$ esto se une con este ramo..." (Sujeto 7)

Los estudiantes refieren que a través del 'estudio de casos' han aprendido a ver las cosas concernientes a la práctica clínica, con un 
prisma más holístico.

“...nos acercan a la realidad de lo que es realmente una persona en un hospital... hay que ver todo lo que... igual la familia, todo eso..., no tan sólo cuando vamos a la práctica vamos a ver a tal paciente por la enfermedad que tiene, es el todo el que nos sirve y ese caso, con ese trabajo nos ha ayudado harto porque es la realidad que uno va a ver" (Sujeto 17)

“...nos ayuda porque en la primera práctica no solamente nos vamos a basar en técnicas sino que como ya lo estamos haciendo...psicología, no vamos a llegar y hacer una técnica y dejar al paciente ahí, sino que ver todos los problemas que pueda tener,..." (Sujeto 3)

Los estudiantes reflejan el valor que le otorgan a sus prácticas como espacios de formación.

\section{Subcategoría 2.3.2 Aplicación de los apren-} dizajes al futuro laboral: Con el 'estudio de casos', los estudiantes visualizan el rol que debe asumir el profesional de Enfermería.

“...porque uno se está transfiriendo a otro escenario donde tiene que pensar como si ya estuviera listo para trabajar con personas" (Sujeto 8)

“... este aprendizaje que se nos entregan, que es un completo para poder llevarlo a cabo cuando se trata de una persona viva, que necesita de profesionales capacitados" (Sujeto 16)

"Como lo que vamos a hacer en definitiva cuando egresemos. Estudiar un caso, de una persona, de una familia..." (Sujeto 11)

Si un conocimiento es aplicado en un contexto diferente a aquel en que se aprendió, significa que efectivamente ha sido internalizado, es decir, ha sido reconstruido y apropiado.
Subcategoría 2.4. Autonomía en el aprendizaje: Los estudiantes perciben que el 'estudio de casos' los impulsa a tener que realizar actividades para adquirir los conocimientos.

“...Porque nos deja a nosotros buscar nuestra información, de integrar, de conocer,... es algo activo. No sólo que la profesora nos entregue el conocimiento" (Sujeto 9)

"... uno mismo como que se va inventado cosas, o de hacer, o vamos para allá a un consultorio a averiguar más, de comprar quizás algún libro que tenga que ver con la enfermedad" (Sujeto 6)

Algunos estudiantes prefieren esta forma de trabajar, que estimula la búsqueda activa de los conocimientos, en contraposición a recibirlos inactivamente.

"Estamos acostumbrados a puro recibir la información en las clases,... Nos pasan las clases, las leemos, pero en ningún momento comenzamos, nosotros, a redactar las ideas que tenemos en la cabeza" (Sujeto 4)

“... se tiene las presentaciones de las clases, se puede estudiar las clases y no busca más allá... acerca de los temas" (Sujeto 3)

Categoría 3. Mediación docente: Un vínculo referido por los estudiantes, para guiar el trabajo académico del 'estudio de casos', es el docente.

“...pudimos aprender de nuestra tutora la cual fue de gran ayuda en la elaboración y desarrollo de este caso" (Documento de reflexiones, grupo 1)

“...estuvo dispuesta a trabajar con nosotros, aclarándonos todas las dudas... La disposición de ella junto con su entusiasmo fue crucial para mí. Gracias a ella yo entendí la importancia del caso integrado y cambié mi actitud hacia él" (Sujeto 2) 
“... así que nos orienta en las tareas... Nos entrega ánimo para concluir este proceso lo mejor posible" (Documento de reflexiones, grupo 4)

Los alcances de la investigación no permiten establecer con precisión el valor que posee, en el proceso de conducción del método, el profesor. Sin embargo las declaraciones de los estudiantes permiten vislumbrar el valor que tiene quien guía el trabajo cooperativo; a veces guía, a veces conductor, a veces motivador, a veces evaluador.

Categoría 4. Formación profesional: Los estudiantes reconocen que el 'estudio de casos' aporta en su 'formación profesional'.

“... todo lo aprendido... entrega seguridad para seguir avanzando en este camino que nos llevará a ser profesionales de la salud, que nos llevará a ser Enfermeros" (Documento de reflexiones grupales, grupo 4)

“... y como le dije me sirve mucho para adelantar camino, o sea no voy a llegar y que hago dónde comienzo, en ese sentido tengo una buena base" (Sujeto 7).

Los alumnos notan que con esta tarea están desarrollando algunas características.

"Creo que este trabajo me ayudará a conseguir una gran gama de características, tales como, responsabilidad, trabajo en equipo, conocimientos, capacidad para investigar, imaginación y pensamiento crítico,..." (Sujeto 4)

“...como a aprender a indagar más” (Sujeto 3)

"Entonces eso ayuda en el tino de poder realizar una acción, en la eficacia, en el tiempo de respuesta. ...Beneficia el futuro desarrollo y desenvolvimiento profesional" (Sujeto 8)

Subcategoría 4.1. Desempeño profesional: El valor que los estudiantes le conceden al 'estudio de casos', es sentir que están siendo preparados para cumplir con un buen 'desempeño profesional'.

"Como futuros enfermeras/os, debemos reorientar nuestro enfoque biomédico no sólo a la enfermedad y su tratamiento, sino más bien a una atención integral para las personas y dar un mejor manejo en salud" (Documento de reflexiones, grupo 3)

"Yo creo que así vamos a ser mejores profesionales" (Sujeto 3)

\section{DISCUSIÓN Y COMENTARIO}

Los aprendices que participaron en este estudio revelaron que, mediante la estrategia 'estudio de casos', experimentaron un proceso fundamental en su formación como profesionales de Enfermería. De acuerdo al objetivo que guió esta investigación, el cual fue analizar las percepciones de estudiantes de Enfermería sobre el 'estudio de casos', utilizado en clases, como estrategias de aprendizaje, se pudo constatar que la educación no está circunscrita tan sólo al ámbito de los saberes y procedimientos. Confluyen también otros elementos vinculados a lo afectivo y relacional (valores y actitudes), que suman grandes desafíos a la sociedad, a las personas, pero fundamentalmente a quien cumple la labor de formar. Tal como los educandos lo expresaron, desarrollar un 'estudio de casos' en un equipo significó identificar sus fortalezas para ponerlas a disposición del grupo y reconocer sus debilidades para pedir ayuda. $\mathrm{Al}$ respecto Zabala (16) señala que los grupos de trabajo deben desarrollar la conciencia de diversidad, solidaridad y apoyo ante la diferencia (16). Los estudiantes conceden un valor al logro de la competencia 'trabajo en equipo colaborativo', cuyo desarrollo representa una preocupación académica para dar respuesta profesional en escenarios laborales diversos y complejos. Esto se reafirma con la 
idea de que en la conformación de un equipo influyen también factores relacionados con algunas habilidades sociales (ayuda mutua, respeto, tolerancia y diálogo) (17). A lo anterior se podría agregar que el liderazgo constituye una competencia valiosa, necesaria de perfeccionar durante la formación de los estudiantes de Enfermería. La profesión ofrece en su ejercicio un lugar a esta capacidad, tanto en los equipos de trabajo como a nivel de la comunidad que atiende. El liderazgo en la formación de un estudiante de Enfermería da valor al ejercicio de la profesión. Podría mejorar la salud del individuo o la familia, si la enfermera/o les ayuda a tomar decisiones para establecer y conseguir objetivos que aumenten su bienestar (18). Se rescata la importancia de reforzar, en atención a lo señalado por los estudiantes, algunas de las características de un líder, como disponibilidad a los requerimientos del grupo; capacidad para organizar y guiar el trabajo; dedicación y esfuerzo para llevar a cabo la tarea. Esto concuerda con lo que Ribbens menciona, en el sentido de que el mayor desafío es que los grupos busquen el liderazgo (6).

Otro elemento que surge de la investigación es la presencia de un profesor que apoye la experiencia pedagógica de trabajar con casos. Los estudiantes reconocen el sustento pedagógico brindado por el docente, evidenciado por la orientación, para llevar a cabo la tarea, su motivación, disponibilidad, entrega y entusiasmo. Allen y Razvi (19) afirman que la influencia del profesor, como facilitador, ha de tenerse en cuenta si se espera que los estudios de casos promuevan un cambio efectivo en los estudiantes. Una condición del método 'estudio de casos', es la capacidad del profesor para conducir la discusión, ayudar a los estudiantes a realizar un análisis más agudo e inducirlos a esforzarse para obtener una comprensión más profunda (20). Otros autores (21) asienten que: “...el conocimiento no puede venir impuesto por expertos sino que tiene que ser producido por medio de la interacción entre profesorado y alumnado".
El 'estudio de casos' también representa para los estudiantes la adquisición de conocimientos, que estiman como profundos, integrados, permanentes, fáciles de replicar. Es necesario que las actividades de enseñanza permitan la integración entre diferentes contenidos y faciliten la comprensión de una realidad que no es compartimentada. Tal es el caso de los problemas de cualquier tipo, en las que los diferentes contenidos de distintas disciplinas son necesarias para su resolución o comprensión (16). Conocimientos que los estudiantes también aprecian como significativos en cuanto se conectan a lo que ya conocen, a lo que palpan en situaciones reales y semejantes en sus prácticas, identificando un rol al que van enfrentando con seguridad y con una base sólida sustentada por el logro de esos aprendizajes. En este aspecto ellos mismos otorgan sentido a los conocimientos adquiridos a través de este método, en la medida que les ayuda a resolver una problemática. El asunto ya no es memorizar lo que dice un texto, sino más bien hacerlo suyo, construirlo, darle utilidad. Es habitual que la mayoría de los estudiantes no utilizan las estrategias adecuadas, reduciendo su aprendizaje a prácticas de memorización y repetición sobre los conocimientos que le transmite el profesor y los textos que utiliza (22); el 'estudio de casos' surge con la intención de romper con esa actitud conveniente y cómoda, pero poco efectiva cognitivamente. La resolución de un problema real busca cambiar el rol pasivo del estudiante, receptor de la información, por un sujeto activo que elabora los contenidos (23). Vinculado a lo anterior, en un artículo sobre la enseñanza de las ciencias, Herreid describe y pondera algunas percepciones de profesores sobre el impacto del uso del 'estudio de casos' como método de aprendizaje, señalando que el 95\% de los profesores expresó que los estudiantes tomaron un papel más activo en el proceso de aprendizaje, y que el 59\% señaló que los alumnos tenían más probabilidades de hacer estudios independientes fuera del aula (5). 
Se dice que en la concepción constructivista, el aprendizaje se logra de una manera activa por parte de los estudiantes, puesto que deben construir, modificar, enriquecer y diversificar sus esquemas de conocimiento acerca de los contenidos a los que les otorga significado y sentido cuando los aprende (24). Además se constató que durante el transcurso del trabajo, los estudiantes han desarrollado importantes cambios en su forma de aprender, transformándose en aprendices activos que toman sus propias decisiones y que construyen aprendizajes útiles para poder aplicarlos a situaciones actuales y que posiblemente enfrentarán en su futuro laboral. $\mathrm{Al}$ respecto, Román opina que: "Aprender a aprender se puede entender como el procedimiento personal más adecuado para adquirir un conocimiento. Esto puede ser enseñado e indica una actitud procedimental a un problema determinado" (25).

Ya lo han experimentado en sus prácticas clínicas, a las que identifican como un espacio de formación, en donde han descubierto y puesto en uso lo que han aprendido con el 'estudio de casos'. Relacionado a esto, Beltrán indica que: “... la capacidad del sujeto para trasladar los conocimientos adquiridos a contextos, o situaciones nuevas, es la esencia del verdadero aprendizaje..." (2).

Por esta razón es importante investigar si los estudiantes son capaces de abstraer los principios generales del 'estudio de casos', aplicarlos y transferirlos a otras situaciones (26). Por otra parte, otros autores (27) concluyen que esta técnica permite una mayor interiorización de los conocimientos de la disciplina.

Con todo, resulta que el método posibilita, por una parte, el aprendizaje activo $y$, por otra, un aprendizaje que se torna significativo; sin duda que ambas premisas son paradigmáticas en la didáctica educativa de hoy.

A la luz de estos elementos, el método se constituye en un sitio de confluencia de los saberes, ahora salen de su parcialidad, irrumpen de los libros, cobran vida, se hacen tan- gibles, se hacen reales, útiles en su aplicación, con sentido de mediación para resolver posibles problemas. Siendo así se podría anticipar el sitio o actividad en que se establece la real y verdadera formación de un profesional de Enfermería. Con el 'estudio de casos' el interés del alumno en el aprendizaje aumenta (20). Para Camille (28) el 'estudio de casos' hace al proceso del aprendizaje científico más genuino y riguroso, dando vida al aprendizaje de la clase y ayudando a los alumnos a entender los desafíos sociales. Resulta revelador certificar que el 'estudio de casos' es una herramienta útil en desarrollar competencias para el plano profesional. Es un método que anticipa el análisis y diagnóstico de una situación de salud, que ayuda a estructurar un plan de solución en un ambiente de simulación del actuar como profesional, que implica la toma de decisiones.

En otro ámbito de este informe, una de las limitaciones para desarrollar la investigación fue el poco tiempo con el que contaron los estudiantes para cumplir con la actividad metodológica, relacionada con el quehacer del resto de sus asignaturas.

En cuanto a las implicancias prácticas, los hallazgos de esta investigación podrían servir como referente para el diseño de programas de capacitación a los docentes, con el propósito de incentivar la utilización del método, producir mejoras en la fase de planificación y orientar su implementación para enriquecer y actualizar sus actividades.

Se sugiere que, para futuras investigaciones, se puedan realizar trabajos de enfoques cualitativos con diseños de reflexión-acción, acerca de las perspectivas procedentes de los docentes que estén utilizando este método en sus actividades curriculares, pues: “... los docentes también son responsables de los significados que se atribuyan a los componentes del currículum y a las formas de desarrollarlos" (29).

En definitiva, se reafirma la necesidad que docentes universitarios, que participan en la formación de estudiantes de Enfermería, 
lleven a sus aulas estrategias que promuevan la construcción de aprendizajes prácticos, significativos, que constituyan un nexo entre la teoría y la práctica, que en su continuidad se transformen en una base sólida para un venidero y óptimo desempeño, en ambientes laborales en donde se exige que los profesionales de Enfermería posean competencias que les permita ser facultativos integrales, líderes, proactivos, que aporten soluciones creativas, concretas y trascendentes a los problemas de salud.

Probablemente el 'estudio de casos' no ofrece a todos los académicos iguales opciones para el desarrollo de sus contenidos. Con todo, sí ha dado muestras claras de constituirse en una herramienta didáctica interesante y sin duda con nítida valoración por parte de los estudiantes de Enfermería, quienes a la postre serán los únicos evaluadores de las estrategias utilizadas en su formación, criterios que ellos fundarán en virtud de su desempeño en el escenario laboral, ámbito concreto desde el cual siempre se calificará la acción formadora de la universidad. Es así como Henderson aseguraba que el valor de la educación de la enfermería proviene de la confianza que el individuo desarrolla durante su formación profesional en la universidad (29).

\section{REFERENCIAS}

1. Conferencia Mundial sobre la Educación Superior. La nueva dinámica de la educación superior y la investigación para el cambio social y el desarrollo. Organización de las Naciones Unidas para la Educación, la Ciencia y la Cultura, París, 5-8 julio 2009. París: UNESCO; 2010.

2. Beltrán J. Procesos, estrategias y técnicas del aprendizaje. $2^{a}$ ed. Madrid: Editorial Síntesis; 1998.

3. López A. Iniciación al análisis de casos. Una metodología activa de aprendizaje en grupos. Bilbao: Ediciones Mensajero S.A.; 1997.

4. Lewis E. Education based on topics or issues in science education. ActionBioscience.org. American Institute of Biological Sciences [Internet]; 2003 Sept [citado 15 septiembre 2006]. Disponible en: http://www.actionbioscience.org/esp/ rducation/lewis.html

5. Herreid CF. Using case studies to teach science. ActionBioscience.org. American Institute of Biological Sciences [Internet]; 2005 May [citado 15 septiembre 2006]. Disponible en: http://www.actionbioscience.org/esp/education

6. Ribbens E. Teaching with Jazz. Using multiple cases to teach introductory Biology. J Coll Sci Teach [Internet]. 2005 Sept [citado 15 septiembre 2006]; 36(2): 1015. Disponible en: http://www.nsta.org/ publications/news/story.aspx?id=52585

7. Gallucci K. Learning concepts with cases. J Coll Sci Teach [Internet]. 2006 Sept [citado 15 septiembre 2006]; 36(2): 16-20. Disponible en: http://www.nsta.org/publications/news/story.aspx?id $=52586$

8. Malkani J, Allen J. Cases in Teacher Education: Beyond Reflection into Practice. [Internet] Annual Meeting of the American Educational Research Association (AERA); 2005 April 11-15; Montreal [citado 15 septiembre 2006]. Disponible en http://eric.ed.gov/PDFS/ED490433.pdf

9. Sadín MP. Investigación cualitativa en educación. Fundamentos y tradiciones. Madrid: McGraw-Hill/Interamericana; 2003.

10. Glaser BG, Strauss A. The discovery of grounded theory: strategies for qualitative research. New York: Aldine de Gruyter; 1967.

11. Huberman MA, Miles MB. Analyse des données qualitatives. Bruxelles: De Boeck; 2003.

12. Corbetta P. Metodología y técnicas de investigación social. 2da ed. Madrid: McGraw-Hill/Interamericana Editores S.A.; 
2007.

13. Tébar L. El profesor mediador del aprendizaje. Santiago: Arrayán Editores S.A.; 2007.

14. Canales M. Metodologías de investigación social. Santiago, Chile: Lom Ediciones; 2006.

15. Hernández R, Fernández C, Baptista P. Metodología de la investigación. 4ta ed. México: McGraw-Hill/Interamericana Editores S.A.; 2006.

16. Zabala A. ¿Qué hace que el alumno y la alumna aprendan los contenidos escolares? En Coll C, Martín E, Mauri T, Miras, M, Onrubia J, Solé I, et al. El constructivismo en el aula. 15a ed. Barcelona: Editorial Graó; 2005. p. 125-161.

17. Díaz-Barriga F, Hernández G. Estrategias docentes para un aprendizaje significativo. Una interpretación constructivista. 2da ed. México: McGraw-Hill/Interamericana Editores S.A.; 2002.

18. Kozier B, Erb G, Bais K, Wilkinson JM. Fundamentos de enfermería. Conceptos, proceso y práctica. 5ta ed. México: McGraw-Hill/Interamericana Editores S.A.; 2002.

19. Allen J, Razvi S. Students' perspectives, levels of epistemological understanding, and critical thinking dispositions related to the use of case studies in an educational psychology course. [Internet] Annual Meeting of the American Educational Research Association (AERA); 2006 April 11; San Francisco [citado 25 septiembre 2006]. Disponible en http://eric.ed.gov/ PDFS/ED491666.pdf

20. Wassermann S. El estudio de casos como método de enseñanza. Buenos Aires: Amorrortu Editores S.A.; 1994.

21. Magalef L, Canabal C, Iborra A. Transformar la docencia universitaria: una propuesta de desarrollo profesional basada en estrategias reflexivas. Perspectiva Educacional. 2007; 48: 73-89.

22. González D, Díaz Y. La importancia de promover en el aula estrategias de aprendizaje para elevar el nivel académico en los estudiantes de Psicología. Revista Iberoamericana de educación [Internet]. 2006 [citado 25 septiembre 2007]: 40:1. Disponible en: http://www.rieoei.org/ investigation/1379Gonzalez.pdf

23. Arberzú MI, Ortega V. Teoría y práctica del sistema modular en la Universidad Autónoma Metropolitana, Unidad Xochimilco. Perspectiva Educacional. 2006; 47: 33-58.

24. Onrubia, J. Enseñar: crear zonas de desarrollo próximo e intervenir en ellas. En: Coll, C, Martín E, Mauri T, Miras M, Onrubia J, Solé I et al. El constructivismo en el aula. 15ta ed. Barcelona, España: Editorial Graó; 2005. p. 65-100.

25. Román, M. Aprender a aprender en la sociedad del conocimiento. Teoría para la práctica. 2da ed. Santiago, Chile: Arrayán Editores S.A.; 2006.

26. Lundeberg MA, Yadav A. Assessment of Case Study Teaching: Where Do We Go From Here? Part II. J Coll Sci Teach 2006; 35(6), 8-11, 13.

27. Gómez-Sánchez E, Dimitriadis Y, Asensio-Pérez JI, Rodríguez-Cayetano M, Bote-Lorenzo ML, Vega-Gorgojo G. Application and evaluation of the use of case studies in courses of Telematics Engineering. En: IV Jornadas de Ingeniería Telemática, JITEL; 2003 September; Gran Canaria, Valladolid: Universidad de Valladolid, Departamento de Teoría de la Señal y Comunicaciones e Ingeniería Telemática; 2003. p. 503-510.

28. Camille P. Case Studies Add Value to a Diverse Teaching Portfolio in Science Courses. J Coll Sci Teach [Internet]. 2006 Oct [cited 2006 Sept 28]: 36(2): 31-37. Available from: http://www.nsta.org/publications/news/story.aspx?id=52689

29. Gimeno Sacristán J. El currículo: una reflexión sobre la práctica. $8^{\mathrm{a}}$ ed. Madrid: Ediciones Morata, 2002. 